\title{
Identification of active ingredients in Wuzhuyu decoction improving migraine in mice by spectral efficiency association
}

\author{
XUEQIANG PAN ${ }^{1 *}$, MANYUAN WANG $^{1 *}$, YANCHUAN WU $^{2}$, XURAN LU $^{1}$, YAWEN SHANG $^{1}$, \\ YONGSONG XU ${ }^{1}$, YONGSONG ZHAI ${ }^{1}$, JING LI $^{1}$, ZHAOXIA LI $^{1}$ and MUXIN GONG ${ }^{1}$ \\ ${ }^{1}$ Beijing Key Lab of TCM Collateral Disease Theory Research, School of Traditional Chinese Medicine, \\ Capital Medical University; ${ }^{2}$ Central Laboratory, Xuanwu Hospital of Capital Medical University, \\ Beijing 100069, P.R. China
}

Received May 17, 2014; Accepted February 5, 2015

DOI: $10.3892 / \mathrm{mmr} .2015 .3506$

\begin{abstract}
Wuzhuyu decoction is a traditional Chinese medicine used for the effective treatment of migraines, termed 'Jueyin headache', in China. However, there have been few investigations to clarify the composition of Wuzhuyu decoction for the treatment of migraines. In the present study, 10 types of Wuzhuyu decoction were analyzed by chromatograms. 5-hydroxytryptamine (5-HT)-depletion mouse models of migraine were prepared by subcutaneous injection of reserpine and placement of autologous blood clots in the cerebral cortex. The levels of 5-HT, noradrenaline (NE), dopamine (DA), nitric oxide (NO) and nitric oxide synthase (NOS) in the brain tissues and sera of the mice were determined. The ingredients and pharmacodynamic indices of the Wuzhuyu decoctions were analyzed using spectral efficiency association by partial least squares regression. The levels of 5-HT, NE and DA in the mouse brain tissues were reduced to $337.785 \pm 84.504$, $171.173 \pm 65.172$ and $242.075 \pm 158.621 \mathrm{mg} / \mathrm{g}$ brain tissue, respectively. The level of $\mathrm{NO}$ in the brain tissues increased to $0.425 \pm 0.184 \mu \mathrm{mol} / \mathrm{g}$ protein and the activities of NOS in the brain tissues and sera increased to $0.719 \pm 0.477 \mathrm{U} / \mathrm{mg}$ and $50.688 \pm 8.132 \mathrm{U} / \mathrm{ml}$, respectively. Regarding the ingredients of the Wuzhuyu decoction, those with significant regression coefficients were ginsenoside-Rg1, Re, Rb1, rutaevine $(\mathrm{Rv})$, limonin (Li), evodiamine (Ev), rutaecarpine $(\mathrm{Ru})$ and substance X (awaiting identification). Rg1, Re, Rb1, Rv, Li,
\end{abstract}

Correspondence to: Dr Muxin Gong, Beijing Key Lab of TCM Collateral Disease Theory Research, School of Traditional Chinese Medicine, Capital Medical University, 10 Xitoutiao, Beijing 100069, P.R. China

E-mail: muxingong54@yeah.net

${ }^{*}$ Contributed equally

Key words: Wuzhuyu decoction, spectral efficiency relationship, 5-hydroxytryptamine depletion migraine mouse model, active ingredient
$\mathrm{Ev}, \mathrm{Ru}$ and $\mathrm{X}$ in the Wuzhuyu decoction were observed to yield the pharmacological effects, whereas Rb1, Rv and Ev were important in index improvement.

\section{Introduction}

A migraines is a multifactorial primary headache disorder, which is characterized by severe unilateral throbbing pain (1). Although its pathogenesis remains to be fully elucidated, progress has been made. Vasogenic, neurogenic and trigeminovascular theories have been suggested (2), changing the understanding of migraines from a vascular disease to a widely accepted neurovascular disease. Several animal models have been used in investigations of pathogens and the development of novel drugs, including a contracting model of the carotid arteriovenous anastomosis and the external carotid artery, and models of cortical spreading depression, neurogenic dural inflammation, nitroglycerin and the depletion of 5-hydroxytryptamine $(5-\mathrm{HT})(3,4)$, which have revealed different pathophysiological characteristics of migraines. Previous studies have found that the signs and symptoms of the nitroglycerin and 5-HT depletion animal models are similar to traditional Chinese medicine (TCM) syndromes, suggesting that these animal models are applicable for TCM investigations. The signs and symptoms of the model of nitroglycerin are similar to those of the hyperactivity of liver-yang and the upward disturbance of wind-fire in migraines, whereas the signs and symptoms observed in the model of 5-HT depletion are similar to those observed in colds, blood stasis and yang deficiency in migraines (5). Therefore, according to the Wuzhuyu decoction characteristics of warming middle-jiao and tonifying deficiency, models of 5-HT depletion were selected for pharmacodynamic investigations in the present study.

At present, triptans, corticosteroids, non-steroidal anti-inflammatory drugs and anti-epileptic drugs are predominantly used for the treatment of clinical migraines, however, the application of these drugs can cause several side effects (6). Therefore, investigations into the use of TCM treatments for migraines has received increasing attention. Evodia, ginseng, ginger and jujube are four commonly used TCMs, contained in the Chinese Pharmacopoeia (7). 
Evodia contains a variety of ingredients, including alkaloids, limonoids and flavonoids (8). Previous pharmacological studies have demonstrated that evodiamine (Ev) and rutaecarpine $(\mathrm{Ru})$ promote the secretion of catecholamines (9) and Ev inhibits the activity of monoamine oxidase (10); transient receptor potential vanilloid type 1 (TRPV1) promotes the phosphorylation of endothelial nitric oxide synthase (NOS) and induces the generation of nitric oxide (NO) (11); and limonin (Li) has a significant neuroprotective effect (12) and inhibits calcium influx and excessive NO production (13). The predominant chemical ingredients of ginseng are a variety of ginsenosides (14), including Rg1, which can protect dopaminergic (DA) neurons (15), improve NO levels in myocardial tissues and promote myocardial angiogenesis (16) and Rb1, which is involved in increasing the levels of monoamine neurotransmitter (17) and anti-oxidative stress (18). The main chemical ingredients of ginger are gingerols (19), including 6-gingerol (6-Gi), which is involved in inhibiting gastric acid secretion (20) angiotensin converting enzyme activity (21) and the lipopolysaccharide (LPS)-induced production of NO (22). These four herbs constitute a classical Chinese herbal formula, termed Wuzhuyu decoction, and clinical and experimental investigations have confirmed its efficacy in the treatment of migraines (23).

Although the chemical ingredients of Wuzhuyu decoction and its pharmacological effects for the treatment of migraine have been investigated (24), the ingredients of Chinese herbal formulae are complicated, and their chemical composition does not produce traditional pharmacological effects, therefore, the active ingredients in treating migraines remain to be elucidated thus affecting efficacy and quality control. To resolve these issues, previous studies have used the method of serum pharmacochemistry to analyze the chemical composition following intragastric administration of Wuzhuyu decoction (25). This method revealed that the ingredients of Wuzhuyu decoction, including isorhamnetin -3-O- $\beta$-D-glucosyl(6'-1')- $\alpha$-L-rhamnoside (Irs), dehydroevodiamine (Deh), Ev, Ru and ginsenoside Rd (Rd), Rg1, Re, $\mathrm{Rb} 1$, are absorbed into the bloodstream. However, whether these are the active ingredients remain to be fully elucidated. In the present study, 10 specific chromatograms of Wuzhuyu decoction and models of reserpine-induced migraine were established. The 10 ingredients of the Wuzhuyu decoction were analyzed quantitatively and semi-quantitatively, and the levels of monoamine neurotransmitter, NO and the activity of NOS were determined. Finally, correlation analyses between the ingredients of Wuzhuyu decoction and various pharmacodynamic indices were performed using a partial least squares regression (PLSR) method. The effective ingredients of the Wuzhuyu decoction for treating deficiency-cold type migraines were detected to control the quality of Wuzhuyu decoction. Spectral efficiency association was used to determine the effective ingredients of the Wuzhuyu decoction in treating migraines, and those significantly involved in improving the pharmacodynamics indices were determined. PLSR, which is most suitable for Chinese herbal formulae, was applied to determine the spectral efficiency of the Chinese herbal formula. The results demonstrated for the first time, to the best of our knowledge, that Rv exhibited a regulatory effect on monoamine neurotransmitters.

\section{Materials and methods}

Experimental design and location. Spectral efficiency association analysis was performed on the components of the Wuzhuyu decoction to determine the efficacy of the herbal formula.

The investigations, including Wuzhuyu decoction high performance liquid chromatography (HPLC) analysis, preparation of the migraine model and determination of the NOS activity and NO content, were performed at the School of Traditional Chinese Medicine, Capital Medical University (Beijing, China). The determination of neurotransmitters was performed in the Central Laboratory, Xuanwu Hospital of Capital Medical University (Beijing, China). The investigations were performed between February and May 2013.

Drugs and reagents. Chemical reference substances of $\mathrm{Rg} 1$, Re, Rb1, Li, 6-Gi, Ev and Ru were purchased from the National Institutes for Food and Drug Control (Beijing, China) and had purities of $>88.5 \%$. These were used as reference substances for content determination. Limocitrin-3-O- $\beta$-D-glucoside (Lcs) was produced in Beijing Key Lab of TCM Collateral Disease Theory Research (Beijing, China), with a purity of $>95 \%$. An NO assay kit, NOS assay kit and coomassie blue were purchased from Nanjing Jiancheng Bioengineering Institute (Nanjing, China). Reserpine was provided by Guangdong Bangmin Pharmaceutical Co, Ltd. (Jiangmen, China; cat. no. 100509). Sumatriptan succinate tablets were provided by Tianjin Huajin Pharmaceutical Co, Ltd. (Tianjin, China; cat. no. 2F69151) and HPLC grade methanol and acetonitrile were provided by Thermo Fisher Scientific (Waltham, MA, USA).

Instruments. Determination of the content of Wuzhuyu decoction was performed using an Agilent 1100 series HPLC system (Agilent technologies, Santa Clara, CA, USA), which was equipped with a quaternary low-pressure infusion pump, inline degasser, autosampler, column oven and diode array detector (DAD). The determination of the monoamine neurotransmitter content was completed using an ESA HPLC system (IKA, Staufen, Germany), which was equipped with a binary low pressure infusion pump, inline degasser, autosampler, column oven and diode array detector. A Silent Crusher M homogenizer (Heidolph, Germany), 3K15 low temperature refrigerated centrifuge (Sigma-Aldrich, St. Louis, MO, USA) and DV215CD analytical balance (Sartorius, Shanghai, China) were also used.

Experimental animals. ICR mice (SPF grade; $\mathrm{n}=168$ ), at 7-8 weeks old, weighing 28-32 g, were purchased from Beijing Vital River Laboratory Animal Technology Co., Ltd. (Beijing, China; License no. SCXK (Beijing) 2012-0001). The mice were bred in a standard barrier environment, and the breeding rooms maintained at a constant temperature $\left(25 \pm 2^{\circ} \mathrm{C}\right)$ and constant humidity (40-60\%). The mice were provided with nutritional pellet feeds and unlimited access to water. The lighting was maintained on a $12 \mathrm{~h}$ light/dark cycle. All animal procedures were approved by Beijing Capital Medical University Animal Ethics Committee (Beijing, China) and were performed 
Table I. Numbers and sources of four medicinal constituents of Wuzhuyu decoction.

\begin{tabular}{|c|c|c|c|c|c|c|c|}
\hline Evodia (no.) & Source & Ginger (no.) & Source & Jujube (no.) & Source & Ginseng (no.) & Source \\
\hline WZY-1 & Zhejiang & SJ-1 & Hebei & DZ-1 & Sinkiang & RS-1 & Wanliang, Jilin \\
\hline WZY-2 & Zhejiang & $\mathrm{SJ}-2$ & Shandong & DZ-2 & Unknown & RS-2 & Wanliang, Jilin \\
\hline WZY-3 & $\begin{array}{l}\text { Jiangxi } \\
\text { (Zhonghua) }\end{array}$ & $\mathrm{SJ}-3$ & Shandong & DZ-3 & Sinkiang & RS-3 & Fusong, Jilin \\
\hline WZY-4 & $\begin{array}{l}\text { Jiangxi } \\
\text { (Chunzhonghua) }\end{array}$ & SJ-4 & Shandong & DZ-4 & Beijing & RS-4 & Fusong, Jilin \\
\hline WZY-5 & Jiangxi & SJ-5 & Shandong & DZ-5 & Hebei & RS-5 & Fusong, Jilin \\
\hline WZY-6 & Hunan & & & DZ-6 & Shanxi & RS-6 & Jilin \\
\hline \multirow[t]{4}{*}{ WZY-7 } & Guangxi & & & & & RS-7 & Jilin \\
\hline & & & & & & RS-8 & Jilin \\
\hline & & & & & & RS-9 & Jilin \\
\hline & & & & & & RS-10 & Weikang \\
\hline
\end{tabular}

according to the Guidance Suggestions for the Care and Use of Laboratory Animals promulgated by the Ministry of Science and Technology of the People's Republic of China. All animal procedures were approved by the Animal Ethics Committee of Beijing Capital Medical University (Beijing, China).

Medicinal materials. The sources of the medicinal materials used are shown in Table I. The medicinal materials were appraised by Professor Luo Rong of the School of Traditional Chinese Medicine, Capital Medical University, and were used in accordance with the requirements for each medicine in Chinese Pharmacopeia 2010 Edition (7). The compositions and crude drug concentrations of the 10 Wuzhuyu decoctions are shown in Table II.

Preparation of Wuzhuyu decoction. Dry pastes of Wuzhuyu decoction were produced in Beijing Key Lab of TCM Collateral Disease Theory Research (Beijing, China), according to a previously reported technique (25). The crude drugs (280 g) were weighed and two extractions were performed. For the initial extraction, water $(2,000 \mathrm{ml})$ was added to the drugs, which were soaked at room temperature for $30 \mathrm{~min}$, and the extraction duration was $15 \mathrm{~min}$. For the second extraction, $1,600 \mathrm{ml}$ water was added and the extraction duration was $12 \mathrm{~min}$. The extracted solutions were combined following filtration using eight layers of gauze (Piaoan, Xinxiang, China), and the solution was decompressed and concentrated into dry pastes at $65^{\circ} \mathrm{C}$ using a rotary evaporator (Eyela N-1100V-W, Tokyo, Japan). Vaccume drying was perfomed in a VO400 oven (Memmert, Schwabach, Bavaria, Germany) The composition and concentration of each Wuzhuyu decoction are shown in Table II.

Preparation of the standard and test solutions. To prepare the standard solutions, $15.2 \mathrm{mg} \mathrm{Lcs}, 4.4 \mathrm{mg} \mathrm{Ru}$ and $9.5 \mathrm{mg}$ Ev were dissolved in $50 \mathrm{ml}$ methanol as stock solution 1. Subsequently, $16.8 \mathrm{mg} \mathrm{Rg} 1,5.4 \mathrm{mg} \mathrm{Re}, 11.3 \mathrm{mg} \mathrm{Rb1}$ and $9 \mathrm{mg}$ 6-Gi were dissolved in $5 \mathrm{ml}$ stock solution 1 , and $5 \mathrm{ml}$ methanol was added to the volumetric flask of $10 \mathrm{ml}$ to make the reference solution A. Methanol was added to $3.33 \mathrm{ml}$ reference solution A to a volume of $5 \mathrm{ml}$, to produce the reference solution $\mathrm{B}$. The serial dilution of the reference solution is identical.

To prepare the test solution, Wuzhuyu decoction dry paste ( $\sim 3.5 \mathrm{~g}$ crude drug) was used and $20 \mathrm{ml}$ analytical grade methanol was added prior to being treated with ultrasound (KQ500E; Kunshan, Suzhou, China) for $30 \mathrm{~min}$ and filtered. The filtrate was evaporated to dryness and the residue was dissolved in HPLC-grade methanol, $(5 \mathrm{ml})$. The solution was filtered using a $0.45 \mu \mathrm{m}$ filter membrane (Jinteng, Tianjin, China) to determine the contents of the 10 types of Wuzhuyu decoction. All solutions were stored at $4{ }^{\circ} \mathrm{C}$ and they were equilibrated to room temperature prior to measurements.

Chromatographic conditions. A Kromasil 100-5 C18 chromatographic column (250x4.6 mm; $5 \mu \mathrm{m}$; Kromasil, Shanghai, China) was used for analysis, with a detection temperature of $30^{\circ} \mathrm{C}$. The mobile phase consisted of methanol-water-acetonitrile, and the gradient programme is shown in Table III. The elution rate was maintained at $1 \mathrm{ml} / \mathrm{min}$, the DAD detecting wavelength was set to $203 \mathrm{~nm}$ and the chromatograms were recorded. The injection volumes of the reference and test samples were $10 \mu \mathrm{l}$.

Preparation of animal migraine models and drug administration. The mice were randomly divided into 14 groups (12 mice in each group) and experiments were initiated 4 days after acclimatization. From the beginning of the experiment, the delivery mode of the preventative and treatment drugs for mice in each Wuzhuyu decoction group was $0.2 \mathrm{ml} / 10 \mathrm{~g}$ body weight, perfused orally with the appropriate quantity of Wuzhuyu decoction. Each group was administered one of 10 different Wuzhuyu decoctions with different contents of ingredients (Table II). The mice in the model group, sham group and normal control group were perfused orally with an equal volume of distilled water. The mice in the positive drug group were only treated with drugs and they were perfused orally with an equal dose of sumatriptan from the sixth day. The experiment was performed for a total of 20 days. Mouse 


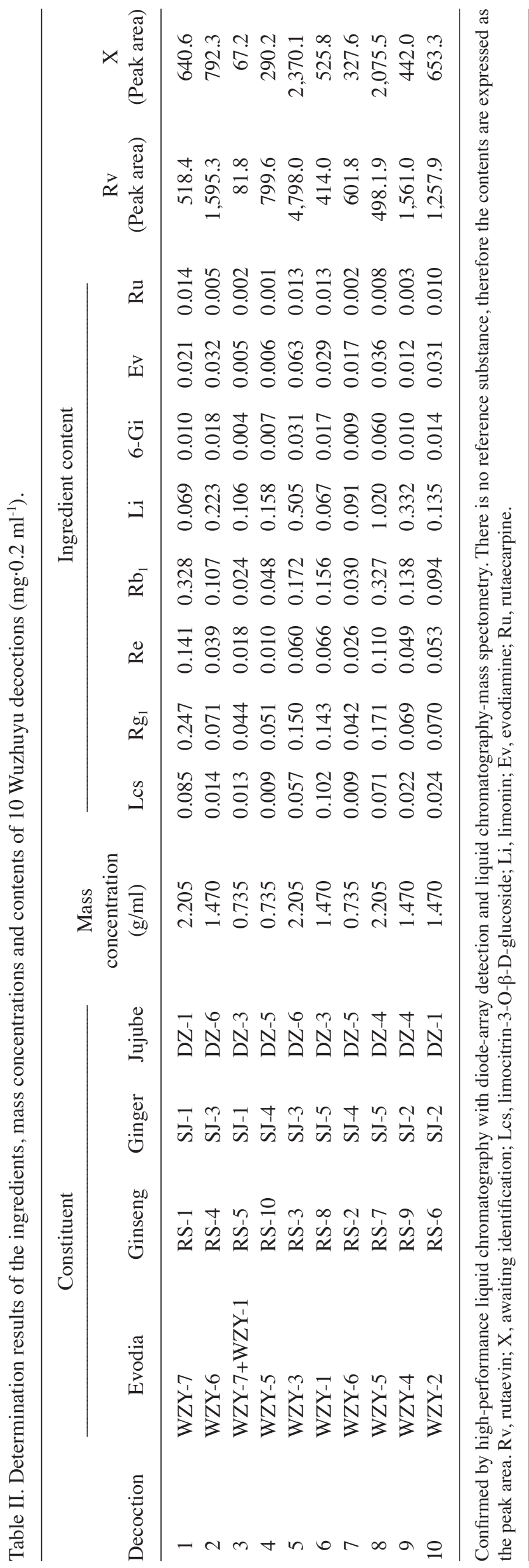


Table III. Gradient program of high performance liquid chromatography analysis.

\begin{tabular}{cccc}
\hline Time (min) & Methyl alcohol (\%) & Acetonitrile $(\%)$ & Water $(\%)$ \\
\hline 0 & 1 & 16 & 83 \\
5 & 1 & 16 & 83 \\
10 & 1 & 19 & 80 \\
45 & 4 & 19 & 77 \\
55 & 4 & 25 & 71 \\
65 & 4 & 34 & 62 \\
110 & 4 & 34 & 62 \\
\hline
\end{tabular}

models were generated between the 6th and 13th day, as described previously (26). The mice in each Wuzhuyu decoction group were injected subcutaneously once with reserpine at $0.17 \mathrm{mg} / \mathrm{kg}$ body weight. The mice were anesthetized using 3\% chloral hydrate (Tianjin Institute of Fine Chemicals retrocessio, Tianjin, China) at $450 \mathrm{mg} / \mathrm{kg}$ body weight on the 14 th day, their scalps were cut and a hole was drilled $2 \mathrm{~mm}$ under the anterior fontanelle, $2 \mathrm{~mm}$ to the right side of the sagittal suture. The autologous blood was collected by cutting the mouse tail, and $2 \mu \mathrm{l}$ autologous blood was injected into the cerebral cortex through the hole at a depth of $1 \mathrm{~mm}$. Following surgery, bone wax (Johnson \& Johnson, New Brunswick, NJ, USA) was used to stop bleeding, penicillin (100 U/mouse; once per mouse following surgery; Nesco Medical Co., Qingdao, China) was used to treat infections, and the wound was sutured. Between the 15th day and 20th day, oral drug administrations were performed, as described above.

Sampling. The mice were anesthetized using 3\% chloral hydrate on the $21^{\text {st }}$ day and $0.5-1 \mathrm{ml}$ mixed blood was extracted by inserting a syringe needle into the apex of the heart, which was transferred into a $1.5 \mathrm{ml}$ centrifuge tube and stored at $4^{\circ} \mathrm{C}$ for $2 \mathrm{~h}$. The blood was centrifuged at 2,296 $\mathrm{g}$ for $10 \mathrm{~min}$ at $4^{\circ} \mathrm{C}$, and the serum was collected and stored at $-20^{\circ} \mathrm{C}$ for the NO content and NOS activity assays. The mice were sacrificed by cervical dislocation, and their brains were removed. The telencephalons were removed by cutting along the needle marks and were stored at $-80^{\circ} \mathrm{C}$ for the NO content and NOS activity assays. The remaining brain tissues were stored separately for the determination of neurotransmitters.

Content determination of monoamine neurotransmitters. The chromatographic conditions used were as follows: ODB-C18 column (4.6x150 mm; $5 \mu \mathrm{m}$; Agilent Technologies) and methanol-buffered saline solution, containing sodium acetate (70 mmol/l), citric acid $(50 \mathrm{mmol} / \mathrm{l})$, EDTA $(100 \mu \mathrm{mol} / \mathrm{l})$ and octane sulfonate $(200 \mu \mathrm{mol} / 1, \mathrm{pH} 4.1)$, which was used for gradient elution. The flow rate was $1 \mathrm{ml} / \mathrm{min}$, the column temperature was $30^{\circ} \mathrm{C}$ and the injection volume was $20 \mu \mathrm{l}$.

Perchloric acid homogenate $(0.4 \%)$ was prepared, as described previously (27), containing $\mathrm{Na}_{2}$ EDTA $(0.5 \mathrm{mmol} / \mathrm{l})$, L-cysteine $(0.83 \mathrm{mmol} / \mathrm{l})$ and 2,5-dihydroxybenzoic acid (internal standard substance; $0.36 \mu \mathrm{mol} / \mathrm{l}$ ). The brain tissues were removed from the $-80^{\circ} \mathrm{C}$ freezer and $0.4 \%$ perchloric acid homogenate was added at $1: 4$ at $4^{\circ} \mathrm{C}$. Tissue homogenization was performed in an ice bath. Brain homogenate was placed in a refrigerated centrifuge at $4^{\circ} \mathrm{C}$ and centrifuged twice at $18,001 \mathrm{x} \mathrm{g}$ for $10 \mathrm{~min}$ each time. The supernatant $(20 \mu \mathrm{l})$ was obtained for the determination of the monoamine neurotransmitter content.

NO content and NOS activity assay. To determine the NO content and NOS activity in the serum, the sera were thawed at room temperature, and $30 \mu 1$ and $100 \mu 1$ were used for determination of NO and NOS, respectively. Each reaction reagent kit (Jiancheng Company, Nanjing, China) was added, according to the manufacturer's instructions, and the reaction was allowed to continue at $37^{\circ} \mathrm{C}$ for a certain time (60 $\mathrm{min}$ for $\mathrm{NO}, 15 \mathrm{~min}$ for NOS and $10 \mathrm{~min}$ for protein meaurements). The absorbance was determined using an ultraviolet spectrophotometer (UV2450; Shimadzu, Kyoto, Japan) at a wavelength of $203 \mathrm{~nm}$ and the absorbance values were recorded to calculate the NO content and NOS activity, according to the manufacturer's instructions.

To determine the NO content and NOS activity of the brain tissues, the brain tissues were thawed at room temperature and physiological saline (Sigma-Aldrich, Shanghai, China) at $4^{\circ} \mathrm{C}$ was added at a 1:9 ratio. Homogenization was performed in an ice bath and the brain homogenate was placed in a refrigerated centrifuge at $4^{\circ} \mathrm{C}$, and centrifuged at $574 \mathrm{x}$ g for $10 \mathrm{~min}$. Supernatant (50 and $500 \mu \mathrm{l}$ ) were collected for the determination of NOS activity and NO content. To determine the protein quantities, mouse brain homogenate $(10 \mu \mathrm{l})$ was dissolved in physiological saline to a volume of $1 \mathrm{ml}$ for analysis. Commassie blue (10 ml; Jiancheng Company) was diluted with deionized water to a volume of $50 \mathrm{ml}$. A protein solution of $0.563 \mathrm{~g} / \mathrm{l}$ was used as standard solution and physiological saline was used as a blank. Diluted commassie blue $(3 \mathrm{ml})$ was added to $50 \mu \mathrm{l}$ diluted brain samples, standard substance and physiological saline. Following incubation for $10 \mathrm{~min}$ at room temperature, the absorbance values were recorded at $595 \mathrm{~nm}$ using a UV-spectrophotometer. The protein content in sample was calculated by the equation: protein $(\mathrm{g} / \mathrm{l})=$ $\left(A b_{\text {standard }}-A b_{\text {blank }}\right) /\left(A b_{\text {sample }}-A b_{\text {blank }}\right)$ x $0.563 \mathrm{~g} / 1$. These results were recorded to calculate the NO content and NOS activity using the formula, according to the manufacturer's instructions.

Statistical analyses. The experimental data were analyzed using SPSS 19.0 statistical software (SPSS, Inc., Chicago, IL, USA). Measurement data is expressed as the mean \pm standard deviation. The data conformed to a normal distribution and 
Table IV. Results of the analyses of linearity of calibration curves and sensitivities for eight ingredients in Wuzhuyu decoction.

\begin{tabular}{|c|c|c|c|c|c|}
\hline Ingredient & Regression equation & Regression coefficient (r) & Linear range (mg/ml) & $\mathrm{LOD}(\mu \mathrm{g} / \mathrm{ml})$ & LOQ $(\mu \mathrm{g} / \mathrm{ml})$ \\
\hline Lcs & $y=33742 x-95.59$ & 0.9997 & $0.0190-0.152$ & 2.88 & 9.61 \\
\hline $\operatorname{Rg} 1$ & $y=2737 x+32.69$ & 0.9996 & $0.2106-1.682$ & 9.62 & 32.06 \\
\hline $\operatorname{Re}$ & $y=2999 x-10.01$ & 0.9997 & $0.0679-0.542$ & 7.89 & 26.31 \\
\hline $\mathrm{Rb} 1$ & $y=2460 . x+12.81$ & 0.9995 & $0.1420-1.134$ & 8.44 & 28.12 \\
\hline $\mathrm{Li}$ & $y=6970 x-32.03$ & 0.9996 & $0.0578-1.846$ & 2.38 & 7.95 \\
\hline 6-Gi & $y=66623 x-153.0$ & 0.9996 & $0.0141-0.2582$ & 0.77 & 2.55 \\
\hline $\mathrm{Ev}$ & $y=41984 x-18.26$ & 0.9996 & $0.0115-0.092$ & 0.66 & 2.19 \\
\hline $\mathrm{Ru}$ & $y=75447 x-65.67$ & 0.9997 & $0.0055-0.044$ & 1.22 & 4.08 \\
\hline
\end{tabular}

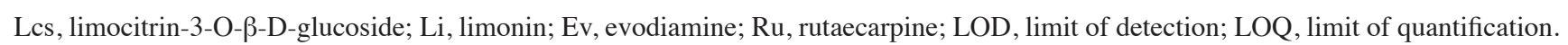

one-way analysis of variance was used to compare between the groups. $\mathrm{P}<0.05$ was considered to indicate a statistically significant difference.

Analytical methods for determining spectral efficiency. Using the PLSR method in SIMCA-P+12.0 Demo, the data on the components and pharmaceutical effects of the Wuzhuyu decoctions were analyzed for spectral efficiency. The principal analysis of the components was performed automatically by the software and, by adding the factor numbers manually, the explanatory abilities of the predominant components for independent variables and dependent variables were $>90 \%$ and the regression coefficients were recorded. The predominant components of Wuzhuyu decoction affecting the pharmacodynamics indices were identified according to the sizes of the regression coefficients.

\section{Results}

Quantitative analysis of the experimental animal model. The ICR mice were randomly divided into 14 groups (12 mice in each group), including a normal control group, sham group, model group, nos. 1-10 Wuzhuyu decoction groups and a positive drug group. Blood was collected from the heart, the sera was obtained and the NO content and NOS activity were determined. In addition, the neurotransmitters, NO content and NOS activity in brain tissue homogenates were determined. Analysis of the final results included 168 mice (12 mice in each group). Characteristics indicative of a successful mouse models were symptoms of continuously decreasing weight and body temperature ( $\geq 3$ days), lassitude, being curled up, inactive and exhibiting limb tremor, and the levels of 5-HT in the brain tissue were significantly decreased. In the present study, the modeling success rate was $90.8 \%$

Investigation methodology. The HPLC analysis conditions, linear associations and sensitivity investigations revealed that the regression equation coefficients of all the components measured were $>0.9995$. The lowest limit of detection and the lowest limit of quantification were $<9.62 \mu \mathrm{g} / \mathrm{ml}$ and $32.06 \mu \mathrm{g} / \mathrm{ml}$, respectively (Table IV). The same mixed reference solution was injected six times continuously and all relative standard deviation (RSD) values of the contents of the measured components were $<3 \%$. Sample preparation was performed repeatedly six times for each Wuzhuyu decoction, the samples were injected and the RSD values of the component contents were $<5 \%$. The same sample of Wuzhuyu decoction was injected five times in $12 \mathrm{~h}(0,3,6,9$ and $12 \mathrm{~h})$, and all the RSD values of the component contents were $<5 \%$, with recovery rates between 97.03 and $106.65 \%$ (Table V). These findings demonstrated that the HPLC method was stable and feasible.

HPLC analyses of the 10 types of Wuzhuyu decoction. Samples of the 10 types of Wuzhuyu decoction and mixed reference substance were examined, according to definite HPLC analysis conditions. The chromatograms of Wuzhuyu decoctions and mixed reference substance were obtained (Fig. 1). A total of 10 common peaks with stable presence and higher response values were observed. Compared with the chromatograms of the mixed reference substances, purity assessment using the DAD detector and LC-MSn structure confirmation revealed that the main ingredients were Lcs (1), ginsenoside Rg1 (2), ginsenoside Re (3), ginsenoside Rb1 (4), rutaevine (Rv) (5), limonin (Li) (6), 6-gingerol (6-Gi) (7), evodiamine (Ev) (8), ingredient X (9) and rutaecarpine ( $\mathrm{Ru})(10)$. From the identified ingredients, eight were analyzed quantitatively using the regression equation (Table IV), and the decoction numbers 5 and 9 were analyzed semi-quantitatively without reference substances as peak areas, as shown in Table II.

Effects of Wuzhuyu decoction on monoamine neurotransmitters in mouse brain tissues and on NO and NOS in brain tissues and sera. The statistical results demonstrated that the contents of 5-HT, NE and DA in the brain tissues of the model mice were reduced to $337.785 \pm 84.504,171.173 \pm 65.172$ and $242.075 \pm 158.621 \mathrm{mg} / \mathrm{g}$, respectively. The NO content in the brain tissue increased to $0.425 \pm 0.184 \mu \mathrm{mol} / \mathrm{mg}$ protein and the NOS activity in the brain tissue and sera increased to $0.719 \pm 0.477$ and $50.688 \pm 8.132 \mathrm{U} / \mathrm{ml}$, respectively. The above indices were compared with those in the normal control and sham groups, and all differences were statistically significant $(\mathrm{P}<0.01)$. Following treatment with the different types of Wuzhuyu, the 5-HT content of the mouse brain tissues were 
Table V. Repeatabilities, stabilities and recoveries of eight ingredients in Wuzhuyu decoction.

\begin{tabular}{|c|c|c|c|c|c|c|c|}
\hline \multirow[b]{2}{*}{ Ingredient } & \multicolumn{2}{|c|}{$\begin{array}{l}\text { Degree of precision } \\
\qquad(\mathrm{n}=6)\end{array}$} & \multicolumn{2}{|c|}{$\begin{array}{l}\text { Repeatability } \\
\qquad(\mathrm{n}=6)\end{array}$} & \multicolumn{2}{|c|}{$\begin{array}{l}\text { Stability } \\
(0-12 \text { h })\end{array}$} & \multirow{2}{*}{$\begin{array}{c}\begin{array}{c}\text { Recovery } \\
(\mathrm{n}=5)\end{array} \\
\text { Mean }(\%)\end{array}$} \\
\hline & $\begin{array}{c}\text { Mean } \pm \mathrm{SD} \\
\left(\mathrm{mg} \cdot \mathrm{ml}^{-1}\right)\end{array}$ & RSD (\%) & $\begin{array}{c}\mathrm{Mean} \pm \mathrm{SD} \\
\left(\mathrm{mg} \cdot \mathrm{ml}^{-1}\right)\end{array}$ & RSD (\%) & $\begin{array}{c}\text { Mean } \pm \mathrm{SD} \\
\left(\mathrm{mg} \cdot \mathrm{ml}^{-1}\right)\end{array}$ & $\operatorname{RSD}(\%)$ & \\
\hline Lcs & $0.2526 \pm 0.0037$ & 1.48 & $0.1502 \pm 0.0051$ & 3.40 & $0.5312 \pm 0.0103$ & 1.95 & 100.13 \\
\hline $\mathrm{Rg}_{1}$ & $0.6290 \pm 0.0062$ & 0.98 & $0.3250 \pm 0.0156$ & 4.80 & $1.0456 \pm 0.0416$ & 3.97 & 100.76 \\
\hline $\mathrm{Re}$ & $0.7372 \pm 0.0159$ & 2.15 & $0.0805 \pm 0.0029$ & 3.63 & $0.2763 \pm 0.0102$ & 3.69 & 104.35 \\
\hline $\mathrm{Rb}_{1}$ & $0.8771 \pm 0.0269$ & 1.66 & $0.3536 \pm 0.0107$ & 3.03 & $1.2522 \pm 0.0574$ & 4.58 & 100.43 \\
\hline $\mathrm{Li}$ & $0.4402 \pm 0.0062$ & 1.41 & $0.7569 \pm 0.0177$ & 2.33 & $2.5837 \pm 0.0259$ & 1.00 & 98.97 \\
\hline 6-Gi & $0.1160 \pm 0.0017$ & 1.46 & $0.0221 \pm 0.0009$ & 3.93 & $0.0744 \pm 0.0035$ & 4.63 & 99.65 \\
\hline $\mathrm{Ev}$ & $0.0611 \pm 0.0010$ & 1.75 & $0.0240 \pm 0.0008$ & 3.50 & $0.0872 \pm 0.0024$ & 2.72 & 97.03 \\
\hline $\mathrm{Ru}$ & $0.0311 \pm 0.0007$ & 2.30 & $0.0112 \pm 0.0006$ & 4.92 & $0.0378 \pm 0.0014$ & 3.64 & 106.65 \\
\hline
\end{tabular}

SD, standard deviation; RSD, relative standard deviation; Lcs, limocitrin-3-O- $\beta$-D-glucoside; Li, limonin; Ev, evodiamine; Ru, rutaecarpine.

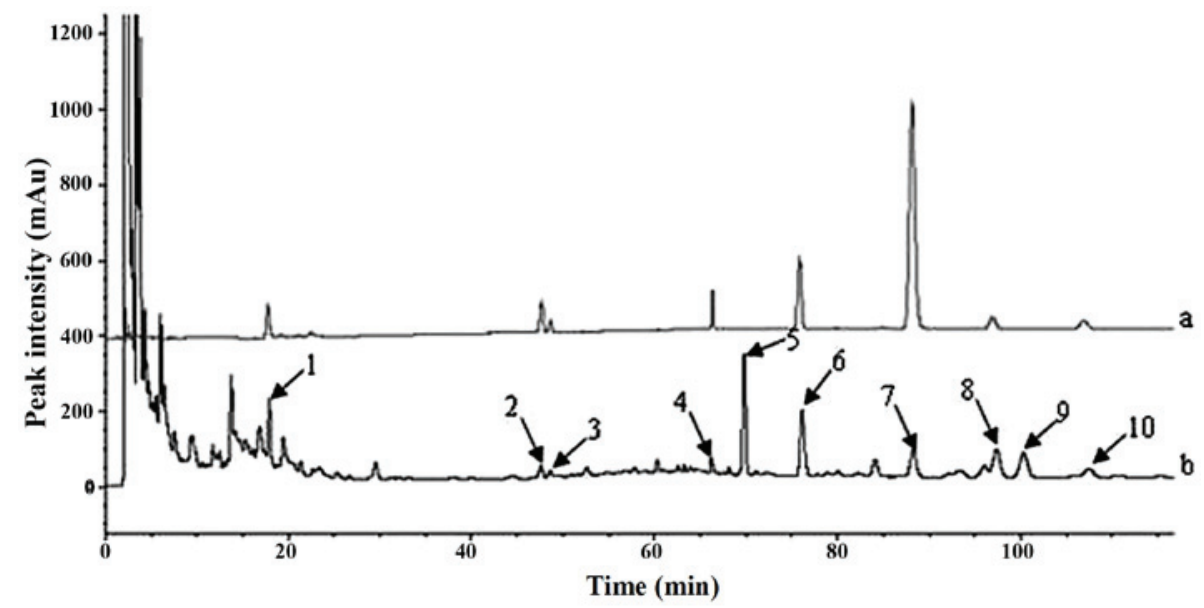

Figure 1. Chromatograms of the (a) mixed reference substances and (b) Wuzhuyu decoction sample. The numbers 1-10 indicated at the peaks indicate an identified ingredient: 1 , limocitrin-3-O- $\beta$-D-glucoside; 2, Rg1; 3, Re; 4, Rb1; 5, rutaevin; 6, limonin; 7, 6-Gi; 8, evodiamine; 9, X (awaiting identification); 10 , rutaecarpine.

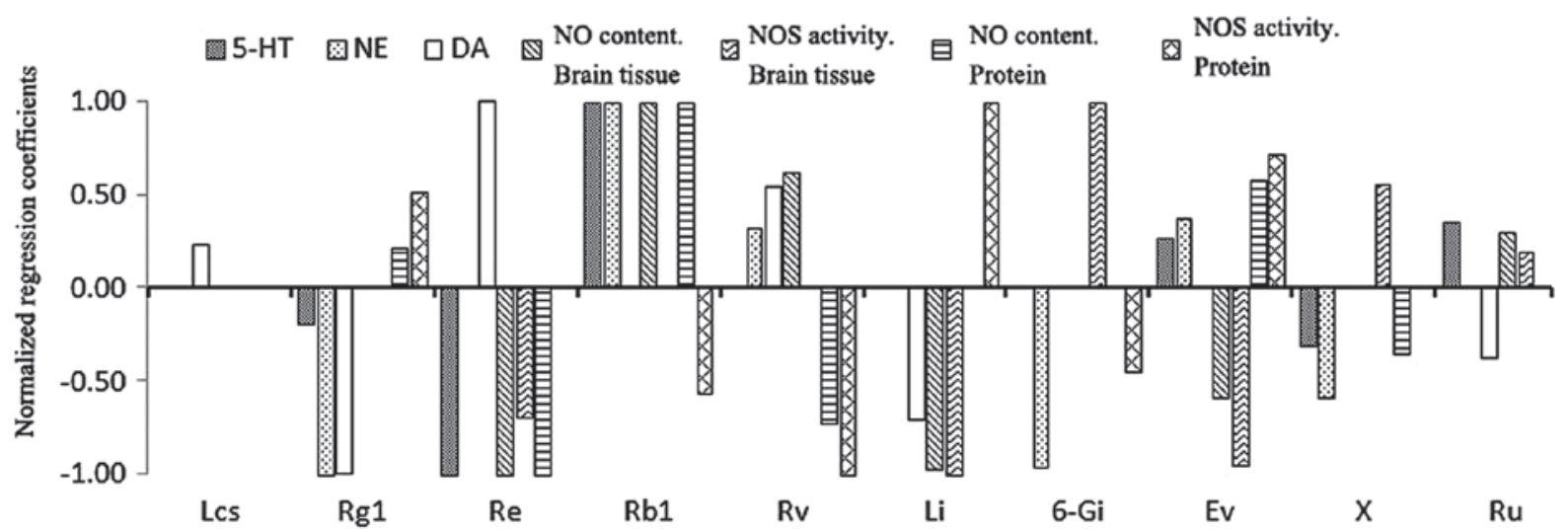

Figure 2. Normalized regression coefficients between the contents of the 10 ingredients in the Wuzhuyu decoctions and the values of pharmacodynamic indices. According to the results in Table VII, the ingredients with the greatest effects on the indices (. with the first three absolute values of the correlation coefficients) were selected, their regression coefficients were normalized and results were obtained. Positive numbers indicated that the ingredient contents had positive correlations with the pharmacodynamic indices and vice versa for negative numbers. The ingredients with significant regulatory effects on four or more pharmacodynamic indicees were Rg1, Re, Rb1, Rv, Li, Ev, X and Ru, of which Rb1, Rv, Ev and Ru had the greatest positive effects. 5-HT, 5-hydroxytryptamine; NE, noradrenaline; DA, dopamine; NO, nitric oxide; NOS, nitric oxide synthase; Lcs, limocitrin-3-O- $\beta$-D-glucoside; Li, limonin; Ev, evodiamine; Ru, rutaecarpine; Rv, rutaevin; Ev, evodiamine; X (awaiting identification). 


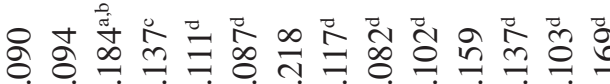

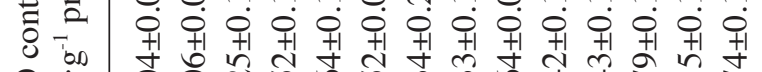

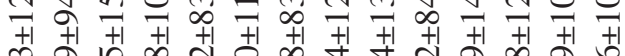
तิ

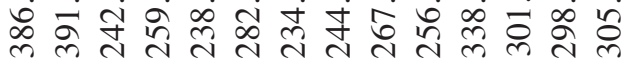

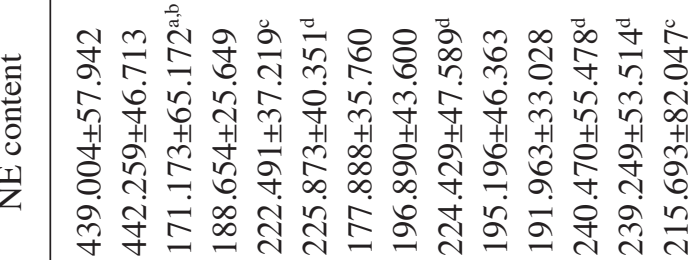

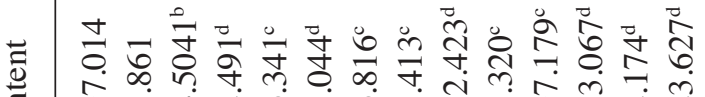
ป

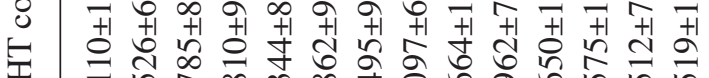

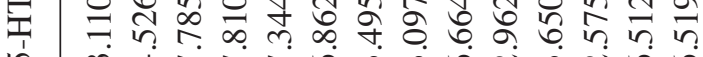

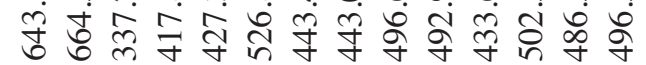

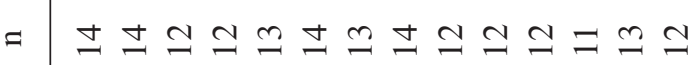
苟 步 远

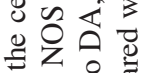
$\exists$ 范

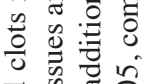
0.0

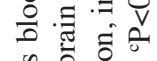

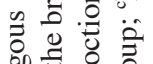
望五过

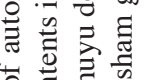

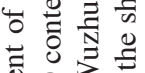

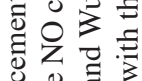
远

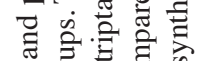

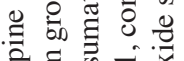

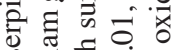

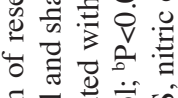

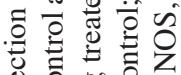

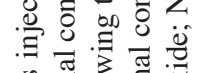


Table VII. Regression coefficients of partial least squares regression between the contents of the 10 Wuzhuyu decoction ingredients and the values of the pharmacodynamic indices.

\begin{tabular}{|c|c|c|c|c|c|c|c|}
\hline Ingredient & $5-\mathrm{HT}$ & NE & DA & $\begin{array}{c}\text { Brain NO } \\
\text { content }\end{array}$ & $\begin{array}{c}\text { Brain NOS } \\
\text { activity }\end{array}$ & $\begin{array}{c}\text { Serum } \\
\text { NO content }\end{array}$ & $\begin{array}{c}\text { Serum } \\
\text { NOS activity }\end{array}$ \\
\hline Les & -0.490 & -0.103 & 1.827 & 0.507 & 0.142 & -0.827 & -1.031 \\
\hline Rg1 & -0.842 & -1.491 & -6.441 & 3.012 & -0.145 & 0.897 & -2.494 \\
\hline $\operatorname{Re}$ & -4.370 & 0.143 & 7.861 & 5.502 & 1.633 & -2.844 & 0.079 \\
\hline $\mathrm{Rb} 1$ & 4.588 & 2.548 & -2.061 & -8.160 & -0.003 & 4.166 & 2.470 \\
\hline $\mathrm{Li}$ & 1.046 & 0.504 & -4.553 & 5.360 & 2.360 & 0.063 & -4.833 \\
\hline 6-Gi & -0.016 & -1.427 & 1.156 & -0.678 & -2.845 & 0.353 & 1.963 \\
\hline $\mathrm{Ev}$ & 1.236 & 0.956 & 0.441 & 3.223 & 2.230 & 2.409 & -3.439 \\
\hline $\mathrm{Ru}$ & 1.624 & 0.004 & -2.464 & -2.445 & -1.333 & -0.732 & 0.513 \\
\hline $\mathrm{Rv}$ & -0.839 & 0.808 & 4.247 & -5.067 & -0.320 & -2.075 & 4.410 \\
\hline unknown X & -1.353 & -0.877 & -0.233 & -0.357 & -0.560 & -0.994 & 0.972 \\
\hline
\end{tabular}

Positive regression coefficients indicated that the ingredient contents were positively correlated with the pharmacodynamic index. Negative regression coefficients indicated that the ingredient contents were negatively correlated with the pharmacodynamics index. The regression coefficients were combined with the indices of improved migraines, of which the regression coefficients of 5-HT, NE and DA content and serum NO contents were either positive, indicating a positive effect on migraine improvement, or negative, indicating a negative effect on migraine improvement (and vice versa for the NO content in brain tissues and the NOS activity in the brain tissues and sera). 5-HT, 5-hydroxytryptamine; NE, noradrenaline; DA, dopamine; NO, nitric oxide; NOS, nitric oxide synthase; Lcs, limocitrin-3-O- $\beta$-D-glucoside; Li, limonin; Ev, evodiamine; Ru, rutaecarpine; Rv, rutaevin; Ev, evodiamine.

all significantly increased, and NE contents in the brain tissues sera were significantly increased. The DA content was increased compared with the model group, but no statistically significant differences were observed between the groups. The NO contents and NOS activity in the brain tissues and sera were significantly decreased, as shown in Table VI.

PLSR analysis. The contents of 10 ingredients, monoamine neurotransmitter and NO contents, and the NOS activities were analyzed by PLSR, and the regression coefficients of the ingredients against different pharmacodynamics indices were obtained (Table VII). Positive numbers indicated that the ingredient contents had positive correlations with the pharmacodynamics indices, and vice versa for negative numbers. The regression coefficients were combined with the indices of improved migraine, of which, those of the contents of 5-HT, NE and DA and serum NO contents were either positive, indicating positive effects on migraine improvement, or negative, indicating negative effects on migraine improvement, and vice versa for NO content in the brain tissues and NOS activity in the brain tissues and sera. Ingredients with greater impacts on the indices (with the first three absolute values of the correlation coefficients) were selected and, following normalization, images were captured (Fig. 2). Ingredients, which had effects on multiple pharmacodynamics indices $(\geq 4)$ were $\mathrm{Rg} 1, \mathrm{Re}, \mathrm{Rb} 1, \mathrm{Rv}$, $\mathrm{Li}, \mathrm{Ev}, \mathrm{X}$ and $\mathrm{Ru}$, of which those with the most positive effects were Rb1, Ev, Ru and Rv, and those with the most negative effects were $\mathrm{Rg} 1, \mathrm{Re}, \mathrm{Li}$ and $\mathrm{X}$. The contents of the above eight ingredients exhibited regulatory effects on the pharmacodynamics indices, therefore, may be the predominant pharmacodynamic substances of Wuzhuyu decoction in treating migraines. These results were confirmed by rewinding and two pharmacodynamic verifications, which produced reproducible and reliable results.

\section{Discussion}

The pathogenesis of migraines remains to be fully elucidated, and animal models of migraine, which are developed according to the different pathophysiological characteristics, have provided support for investigation of the disease and drug development. In the present study, the migraine model used reserpine, resulting in low levels of 5-HT, and placement of autologous blood clots in the cerebral cortices, stimulated respectively by the reduced 5 -HT (28), and the noxious stimulation of internal and external factors on the meninges during migraine attacks (29). Previous investigations have induced headaches in patients with migraines using a method of subcutaneous injection of reserpine, and the association between catecholamine substances and migraine attacks were investigated by examining the metabolic products of 5-HT, adrenaline (A) and NE, including 5-hydroxyindoleacetic acid and 3-methoxy-4-hydroxy mandelic acid, as indicators. It was observed that reserpine-induced migraine headache attacks are associated with excessive catecholamine metabolism (30), therefore, a disturbance of catecholamine metabolism is important in migraines. In addition, meningeal noxious stimulation activates the primary sensory trigeminal fibers, resulting in the release of calcitonin gene related peptide, and endogenous NO is involved in this process (31). The catecholamines, 5-HT, NE, DA, and vasoactive substances, NO, NOS are associated with the modeling mechanism and can visually reflect the pathophysiological characteristics of models, thus, they are selected as pharmacodynamic indices. Sumatriptan is a triptan drug, which is a highly selective $5-\mathrm{HT} 1 \mathrm{~B} / 1 \mathrm{D}$ receptor 
agonist. It is a first-line drug in the treatment of acute migraine attacks (6) and, since its functional characteristics are consistent with the those of model animals, it is used as a positive control drug.

Few studies have reported the correlation between the predominant ingredients and pharmacodynamic effects of Wuzhuyu decoction, limiting its quality control and development. In the present study, the ingredients of Wuzhuyu decoction were associated with their pharmacodynamic effects for the treatment of migraines using a spectral efficiency association method, to detect the predominent effective ingredients affecting the efficacy of Wuzhuyu decoction. The spectral efficiency association method used in the present study is a novel approach suggested on the basis of fingerprint studies. The fingerprints are combined with efficacy results using mathematical methods, for the quality control of TCMs and identifying effective substances with improved specificity compared with simple fingerprints. However, these investigations use predominantly multivariate regression analysis or multivariate correlation analysis, which are not applicable in sample sizes less than or equal to the number of independent variables, or when there is multicollinearity between independent variables. In these situations, the PLSP method may be selected, the use of which has been limited in previous studies and has only been applied to investigate the formula of a single herb (32).

In the present study, using the spectral efficiency association method, the regression coefficients of the various ingredients in Wuzhuyu decoctions with different pharmacodynamics indices were obtained, and, following normalization, results were obtained (Fig. 2). It was revealed that Rg1, Re, Rb1, Rv, $\mathrm{Li}, \mathrm{Ev}, \mathrm{X}$ and Ru had significant regulatory effects on the majority of the pharmacodynamics indices and, therefore, they may be active substances of Wuzhuyu decoction in treating migraines. Four ingredients, Rb1, Ev, Ru and Rv, had an important positive effect on index improvement. These results provided references for determining the effective substances of Wuzhuyu decoction in treating migraines and in selecting the quality control indices.

At present, the pharmacodynamic indices selected following analysis of spectral efficiency remain relatively simple and do not include deeper pathophysiological changes in migraine attacks, including the gene expression of c-fos, release of endogenous opioid peptides and lack of animal behavioral indices. These issues require consideration and improvement in future investigations. The present study also demonstrated that the effects of the variety of ingredients of a compound on pharmacodynamics indices are complicated. The same ingredient may exhibit different effects on different indices and different ingredients can have a synergistic effect on the same indices. This indicates that, when a variety of ingredients in a compound are important and multiple mechanisms are activated simultaneously, close attention is required on the effects of active constituents and their proportions on their efficacy and safety. Therefore, optimization of the ingredients of Wuzhuyu decoction in treating migraines requires further investigation.

\section{Acknowledgements}

This study was supported by the National Natural Sciences Foundation of China (no. 81473360), the Science and
Technology Development Program of Beijing Municipal Commission of Education (no. KM20121002501) and the Capital Key Research Program of Traditional Chinese Medicine (no. 13ZY02).

\section{References}

1. Headache classification subcommittee of the international headache society. The international classification of headache disorders. Cephalalgia 24: 9-160, 2004.

2. Arulmozhi DK, Veeranjaneyulu A and Bodhankar SL: Migraine: Current concepts and emerging therapies. Vascul Pharmacol 43: 176-187, 2005.

3. Arulmani U, Gupta S, VanDenBrink AM, Centurión D, Villalón CM and Saxena PR: Experimental migraine models and their relevance in migraine therapy. Cephalalgia 26: 642-659, 2006.

4. Supornsilpchai W, Sanguanrangsirikul S, Maneesri S and Srikiatkhachorn A: Serotonin depletion, cortical spreading depression and trigeminal nociception. Headache 46: 34-39, 2006.

5. Wang LL and Fan JP: The use of the model animals with migraine in studying of traditional Chinese medicine. Chin Arch Trad Chin Med 25: 760-762, 2007.

6. Gelfand AA and Goadsby PJ: A neurologist's guide to acute migraine therapy in the emergency room. Neurohospitalist 2: 51-59, 2012.

7. China Pharmacopoeia Committee. China Pharmacopoeia. Vol 1) China Medical Science Press, Beijing, China, pp.8, 21, 93 and $160,2010$.

8. Wang YX, Gong MX, Wang ZM, Zhang QW, Gao HM and Song YF: A summary of the studies on the chemical constituents in plants of Evodia J.R.et G. Forst. Chinese Pharmaceutical Journal 45: 641-646, 2010.

9. Zhao FR, Mao HP, Zhang H, Hu LM, Wang H, Wang YF, Yanagihara N and Gao XM: Antagonistic effects of two herbs in Zuojin Wan, a traditional Chinese medicine formula, on catecholamine secretion in bovine adrenal medullary cells. Phytomedicine 17: 659-668, 2010.

10. Lee SS, Hwang BY, Ro JS and Lee MK: Inhibition of monoamine oxidase by evodiamine. Saengyak Hakhoech 37: 320-323, 2006.

11. Ching LC, Chen CY, Su KH, Hou HH, Shyue SK, Kou YR and Lee TS: Implication of AMP-activated protein kinase in transient receptor potential vanilloid type 1-mediated activation of endothelial nitric oxide synthase. Mol Med 18: 805-815, 2012.

12. Yoon JS, Sung SH and Kim YC: Neuroprotective limonoids of root bark of dictamnus dasycarpus. J Nat Prod 71: 208-211, 2008.

13. Yoon JS, Yang H, Kim SH, Sung SH and Kim YC: Limonoids from dictamnus dasycarpus protect against glutamate-induced toxicity in primary cultured rat cortical cells. J Mol Neurosci 42: 9-16, 2010.

14. Kim DH: Chemical diversity of Panax ginseng, Panax quinquifolium and Panax notoginseng. J Ginseng Res 36: 1-15, 2012.

15. Xu H, Jiang H, Wang J and Xie J: Rg1 protects iron-induced neurotoxicity through antioxidant and iron regulatory proteins in 6-OHDA-treated MES23.5 cells. J Cell Biochem 111: 1537-1545, 2010.

16. Zhang QY, Chen YP, Liu F and Chen X: Ginsenoside-Rg1 promotes angiogenesis in rats with acute myocardial ischemia. Third Military Medical University Journals (in press) Pharmacol Therapeut 20: 482-486, 2012-2013.

17. Lee SH, Hur J, Lee EH and Kim SY: Ginsenoside Rb1 modulates level of monoamine neurotransmitters in mice frontal cortex and cerebellum in response to immobilization stress. Biomol Ther (Seoul) 20: 482-486, 2012.

18. Kim S, Na JY and Song KB: Protective effect of ginsenoside Rb1 on hydrogen peroxide-induced oxidative stress in rat articular chondrocytes. J Ginseng Res 36, 161-168, 2012.

19. Chrubasika S, Pittlerc MH and Roufogalisb BD: Zingiberis rhizoma: A comprehensive review on the ginger effect and efficacy profiles. Phytomedicine 12: 684-701, 2005.

20. Okumi H, Tashima K, Matsumoto K, Namiki T, Terasawa K and Horie S: Dietary agonists of TRPV1 inhibit gastric acid secretion in mice. Planta Med 78: 1801-1806, 2012.

21. Liang C, Yun NY, Jung SW, Kim DS, Lee YJ and Ma JY: Analysis of the ingredients of Guibitang and fermented Guibi-tang and their ability to inhibit angiotensin-converting enzyme. Nat Prod Sci 17: 363-366, 2011. 
22. Hong SS and Oh JS: Phenylpropanoid ester from Zingiber officinale and their inhibitory effects on the production of nitric oxide. Arch Pharm Res 35: 315-320, 2012.

23. Gong MX, Wang YX, Zou ZD and Wang ZM: Regularity of Syndrome and medication of wuzhuyu decoction in modern clinical application. China Journal of Experimental Traditional Medical Formulae 15: 84-86, 2009.

24. Yang XW, Xiao SY, Yang Z, Du LJ, Bi KS, Chen DW, Chen DF, Wang ZM and Zou YH: Studies on chemical constituents of the on chemical constituents of the refined wuzhuyu capsule. Journal of Peking University (Health Sciences) 33: 280-282, 2001.

25. Gong MX, Wang YX, Sun J, Zhang QW and Wang ZM: Dynamic analysis of 10 ingredients of the Chinese herbal compound Wuzhuyu-tang absorbed into rat plasma: Neural Regen Res 6: 2594-2599, 2011

26. Du LJ, Sun H, Li M, Su XL, Wang ZM and Xiao SY: Study of the effect of the refined Wuzhuyu Capsule in a mouse model of migraine. Pharmacology and Clinics of Traditional Chinese Medicine 15: 3-5, 1999.

27. Yang S, Zhang DK, Su ZT: Effect of different proportion and different dosage forms of combination of Chuanxiong rhizoma and Angelicae dahuricae radix on migraine in animal models. Chinese Journal of Experimental Traditional Medical Formulae 17: 225-228, 2011.
28. Curzon G, Barrie M and Wilkinson MI: Relationships between headache and amine changes after administration of reserpine to migrainous patients. J Neurol Neurosurg Psychiatry 32: 555-561, 1969.

29. Nozaki K, Boccalini P and Moskowitz MA: Expression of c-fos-like immunoreactivity in brainstem after meningeal irritation by blood in the subarachnoid space. Neuroscience 49: 669-680, 1992

30. Juhasz G, Zsombok T, Modos EA, Olajos S, Jakab B, Nemeth J, Szolcsanyi J, Vitrai J and Bagdy G: NO-induced migraine attack: strong increase in plasma calcitoningene-related peptide (CGRP) concentration and negative correlation with platelet serotonin release. Pain 106: 461-470, 2003

31. Hibino T, Yuzurihara M, Kanno H, Kase Y and Takeda A: Goshuyuto, a traditional japanese medicine and aqueous extracts of Evodiae Fructus constrict isolated rat aorta via adrenergic and/or serotonergic receptors. Biol Pharm Bull 32: 237-241, 2009

32. Wang HW, Wu ZB and Meng J. Partial least-squares regressionlinear and nonlinear methods. Beijing, National Defense Industry Press, pp.2, 2006. 